\title{
Models of teachers' trusty relations and peculiarities of interactions in educational space: empirical research
}

\author{
Tatiana Skripkina ${ }^{1,2, *}$ \\ ${ }^{1}$ Institute of Psychology L.S. Vygotsky \\ ${ }^{2}$ Russian State University for the humanities, Moscow, Russia
}

\begin{abstract}
The theoretical analysis of home and foreign research shows that teacher's ability to set up trusting atmosphere in educational space is the most important factor of professional success and the guarantee of occupational health. The given research is based on the author's concept that human trust in others is inseparable from self-trust, and the ratio of the level of expression of these two oppositely directed attitudes creates the features of trusting relations between a teacher and his (her) students. The mentioned trusting attitudes are included by the author into the teacher's communicative competence structure. A study of self-trust and trust in others among teachers revealed six different models of expression of trusting relations among teachers a model with high self-trust and trust in others; a model with high level of trust in others and medium self-trust; a model with high level of trust in others and low self-trust; a model with low level of trust in others and high self-trust; a model with low level of trust in others and medium self-trust; a model with low self-trust and low level of trust in others. An analysis of the relationships of trusting relations indicators in each of the identified models was then carried out with such qualities of communicative competence as strategies of behaviour in conflict, communicative tolerance, self-control in communication and empathy. The results showed that each empirical model of trusting relationships inherent to different teachers had different relationships with other indicators of communicative teacher's competence included in the study. The results of the study enabled to obtain six different models of teacher-student interaction.
\end{abstract}

\section{Introduction}

Trust has long been known to be a universal imperative of all types of social relationships. It is trust that turns the fact of impersonal communication into the fact of communication. At present there are many interpretations of trust and, at present, scientists do not see eye to eye about its essence, conditions of generation, and its role in different contexts of interaction.

Our attention was drawn to the problem of teachers` trust in students. In our opinion, it is a key factor of the teacher 's communicative competence when interacting with students. At

\footnotetext{
*Corresponding author: skripkinaurao@mail.ru
} 
present, in both domestic and foreign psychology there is quite a large number of applied research on this problem. In modern foreign and Russian psychology the problem of trust in educational practice and positive relations of a teacher with students in general does not lose its relevance, despite the fact that since the 1990s the study of trust in a variety of contexts has been devoted to a huge number of studies Dontsov A.I., Perelygina E.B., Zotova, O. Y., Bridges S. V. [1] Skripkina T. P.[2], Svetlana N. Kostromina, Svetlana D. Gurieva, Maria M. Borisova, Elizaveta G. Khomyakova. [3], Laura Teodora David, Gabriela Lavinia Nită [4].

Studies on the role and functions of trust in educational practice have increased significantly in recent years. A brief analysis of contemporary English-language studies highlighted the following points. Thus, a study to identify the role of teacher-student relationships in a teacher 's personal development and emotional exhaustion and burnout suggested that the quality of teachers' relationships with students affected both the teacher 's psychological well-being and learning outcomes. The study, based on a transactional model of stress and coping found that positive communication and openness with pupils allows teachers to experience satisfaction with their professional activities, feel competent, and, on the contrary, conflict relationships with students lead to stress and burnout Corbin, Catherine M., Alamos, Pilar, Lowenstein, Amy E., Downer, Jason T., Brown, Joshua L.[5].

Back in 2014, when studying emotional burnout in teachers, A.L.Caruso, showed the relationship between emotional burnout and the level of interpersonal trust with teachers on the one hand, and the relationship between the student 's psychological safety and his(her) level of trust in a teacher, on the other hand [6]. In another study it was also shown that emotional deformations and emotional burnout are related to deformations of trust relations in teachers of pre-school institutions [7].

Thus, many authors have shown that the psychological and professional health of a teacher can be preserved in educational space only on the basis of positive communication and trust.

Another, from our point of view, import study was conducted by Kathy T. Do, João F. Guassi Moreira, Eva H. Telzer which proved the need for psychological support for adolescents related to their self-affirmation. The of the study recorded that support of this kind increases trust in school and in class executives, and improves their behavior [8]. However, the other side of trust must be touched upon. After all, it can be used differently. It is about trust used as a factor of manipulation, it destroys so hardly established trust, as evidenced by the study conducted by Duygu Türker, Ceren Altuntaş and Elisa KonyaBaumbach, Monika C. Schuhmacher, Sabine Kuester, Victoria Kuharev [9, 10]. They have shown that even though trust is a decisive factor in cooperation, it can be used for exploitation and unethical behavior. However, this problem is far less explored than the positive aspects of trust. However, it must be understood that trust used this way leads to conflicts and disruption of relations.

In general, in the English language literature there is a significant amount of research on various aspects of teacher-student relations, in which the idea is drawn that close relationships and support in relationships positively affect both behavior and student performance: Andrea Wullschleger, Ariana Garrote, Susanne Schnepel, Lea Jaquiéry, Elisabeth Moser [11], Tuomo Erkki Virtanen, Grete Sørensen Vaaland, Sigrun Karin Ertesvåg [12], Diane M. Early, Kelly L. Maxwell, Bentley D. Ponder, Yi Pan [13], Karen Aldrup, Uta Klusmann, Oliver Lüdtke, Richard Göllner, Ulrich Trautwein [14], Yaoxin Zhang, Wenxu Song, Zhenlin Tan, Huilin Zhu, Li Yi [15], Laura Teodora David, Gabriela Lavinia Nită [16], Berna Akçinar [17], Maria Keilow, Chunsen Wu, Carsten Obel [18], Idalis Villanuevaa,*, Marialuisa Di Stefanob , Laura Gellesa , Paul Vicioso Osoriaa, Sheree Bensona [19], Rebecca E. Lacey, Snehal M. Pinto Pereira, Leah Li, Andrea Danese [20]. 


\section{The aim of the study}

The carried out analysis shows that, despite a number of studies, we have not been able to find those on the relationship between teacher's personality and professional behaviour strategies with different levels of trust in both ourselves and students. This is the problem that this study focused on. The main purpose of the study was to develop models of communicative competence of teachers based on the factor of the teacher's trust in oneself and others.

Here it is necessary to dwell a little on the research concept of the author. We have developed a holistic concept of personal trusting relations. We will not elaborate on the main elements of this concept. Its summary is contained in an article [21]. It should be noted only that we assume from the principle provision that when a person interacts with another (or others), a new psychological situation arises, where each of the interacting subjects has two psychological attitudes simultaneously: trust in oneself and trust in others. It is the expression of these settings (or a measure of trust in oneself and others) that gives rise to different types of interaction in which trust is a background condition (from friendship to manipulation and dependence). One of the mechanisms of activity regulation is the level, or measure, of the expression ratio of trust in oneself and in the world (or its part which the subject interacts with at each moment of his(her) life).

We assumed that the teacher 's ability to build trusting human relations with other subjects of educational activity and, first of all, with students is the most important professionally communicative competence.

\section{Materials and methods}

Here it is necessary to dwell a little on the research concept of the author. We have developed a holistic concept of personal trusting relations. We will not elaborate on the main elements of this concept. Its summary is contained in an article [21] (Skripkina T.P., 2019). It should be noted only that we assume from the principle provision that when a person interacts with another (or others), a new psychological situation arises, where each of the interacting subjects has two psychological attitudes simultaneously: trust in oneself and trust in others. It is the expression of these settings (or a measure of trust in oneself and others) that gives rise to different types of interaction in which trust is a background condition (from friendship to manipulation and dependence). One of the mechanisms of activity regulation is the level, or measure, of the expression ratio of trust in oneself and in the world (or its part which the subject interacts with at each moment of his(her) life).

A review of the literature on the study of psychological competence of a teacher showed that different authors refer to different qualities as indicators of competence of a teacher. In this regard, as the main indicators of communicative competence we have chosen only those qualities named by all authors developing the problem of communicative competence of a teacher. These qualities include trust in others, self-trust, tolerance in communication, empathy, strategies to deal with conflict, self-control in communication. Based on these indicators, the study model was designed. The study included the following technique:

1. Rotter's Interpersonal trust scale [22]

2. Psychometric method "self-Confidence" by Mark Snyder [23]

3. Tolerance in communication (Boiko's method) [24]

This test contains 9 scales. In the study the indicators of each scale were taken into account:

Scale 1. Rejection or misunderstanding of other person 's identity

Scale 2. Use of oneself as a benchmark in assessing behavior and way of thinking

Scale 3. Categorism or conservatism in assessing other people 
Scale 4. Inability to hide or smooth unpleasant feelings when colliding with uncommunicative qualities of partners

Scale 5. Desire to change, re-educate partners

Scale 6. The desire to fit a partner, make him(her) "convenient"

Scale 7. Inability to forgive others' mistakes, awkwardness, unintentional trouble caused to you.

Scale 8. Intolerance of physical or mental discomfort created by others

Scale 9. Inability to adapt to the character, habits and desires of others

4. Explicit measures of empathy [25]

5. Self controlled case series methods [26]

6. Evaluation of the methods of response in conflict (methodology of K.N. Thomas) [27]

\section{Results}

On the basis of the described ideas, we designed 6 psychological models of teacher's trust, which differed depending on the characteristics of self-trust and trust in others of a teacher (a total of 234 teachers of secondary general education schools participated in the study):

1. High level of self-trust and high level of trust in others $-20.5 \%$

2. High level of trust in others and medium self-trust $-19.2 \%$ of total sample

3. High level of trust in others and low self-trust $-14.1 \%$

4. Low level of trust in others and high self-trust $-20.5 \%$

5. Low level of trust in others and medium self-trust - $16.7 \%$

6. Low self-trust and low level of trust in others - $10.3 \%$

As can be seen from the data obtained, models 1, 2 and 4 are represented most of all in percentage terms.

We then conducted a correlation analysis between confidence indicators and those of others used in the study of techniques. On the basis of the correlation analysis carried out, correlation galaxies of indicators of communicative competence were built, depending on the level of self-trust and trust of others with teachers involved.

As a result of the correlation analysis we have obtained six types of models of modern teachers, based on different ratios of expression of the level of trust in oneself and in other people.

Let's analyse the received models. 


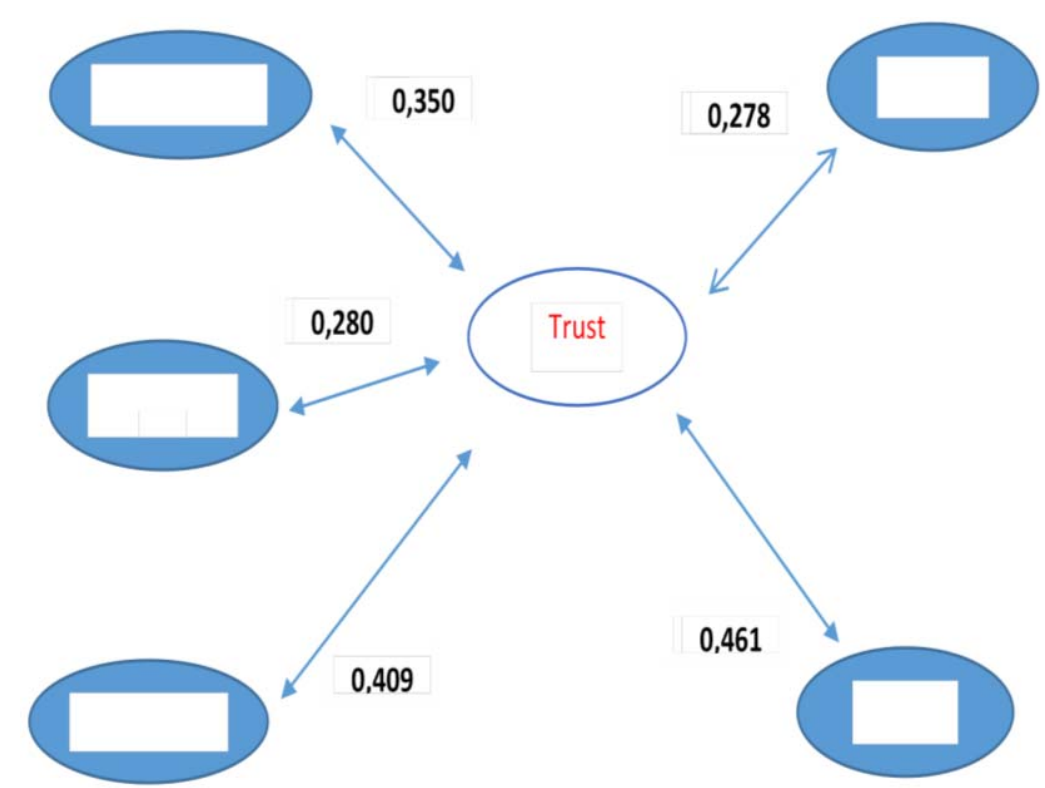

Scale 2 The use of oneself as a benchmark in assessing behavior and way of thinking Scale 5. Desire to change, re-educate partners

Fig. 1. A model with a high level of self-trust and trust in others.

Apparently from the received empirical model, the trust of a teacher highly trusting in himself and in others is significantly interconnected with adaptation strategy and is negatively interconnected with avoidance and compromise strategies, and also with scales of communicative tolerance: the use of oneself as a standard when assessing of behavior and views and the aspiration to change, re-educate partners.

This means that a teacher with high level of trust in oneself and others is not inclined to compromise in conflict situations, he(she) is inclined to adapt in a difficult conflict situation, that is, he(she) does not avoid conflict situations, but chooses a strategy to adapt to a difficult situation. In addition, such a highly trusting teacher does not consider him(her)self a benchmark for others when assessing them and does not seek to re-educate, change others, but allows them to be different.

Apparently, as far as empathy and self-control are concerned, there are no significant connections. It seems to us that a teacher, highly trusting in oneself and others is not inclined to build emotional relationships with people. Thus, a teacher with high level of self-trust and trust in others tries to escape conflicts, he(she) either ignores them or adapts them, as well as shows a high degree of communicative tolerance.

In the total sample of teachers with this ratio of qualities of communicative competence proved to be $19 \%$. 


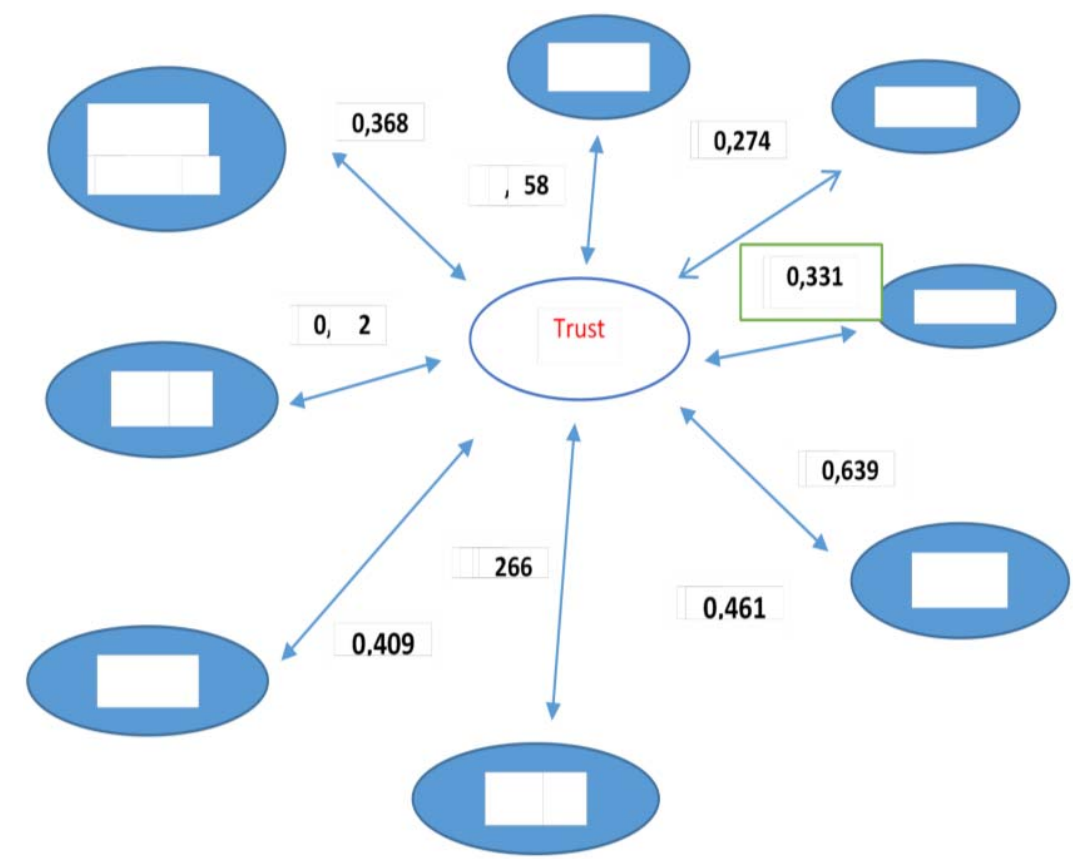

Scale 1. Rejection or misunderstanding of other person 's identity

Scale 2. Use of oneself as a benchmark in assessing behavior and way of thinking

Scale 3. Categorism or conservatism in assessing other people

Scale 4. Inability to hide or smooth unpleasant feelings when colliding with uncommunicative qualities of partners

Scale 5. Desire to change, re-educate partners

Scale 7. Inability to forgive others ' mistakes, awkwardness, unintentional trouble caused to you.

Scale 9. Inability to adapt to the character, habits and desires of others

Fig. 2. A model with high level of trust in others and medium self-trust.

When a teacher trusts in others a little more than him(her)self, we have relationships with a lot more relationships. following indicators. But with the figure showing, all meaningful relationships except empathy are negative:

-rejection or misunderstanding of other person 's identity

- use of oneself as a benchmark in assessing behaviour and way of thinking

- Categorism or conservatism in assessing other people

- Inability to hide or smooth unpleasant feelings when colliding with uncommunicative qualities of partners

- Desire to change, re-educate partners

- Inability to forgive others` mistakes, awkwardness, trouble caused to you inadvertently.

- Inability to adapt to the character, habits and desires of others

So, a teacher able to trust students at a high level tends to show a high degree of empathy and is also characterized by a very high level of communicative tolerance, which is reflected in a high degree of acceptance of students ' individuality, He does not show categorism in assessing children, does not position himself as a benchmark, can smooth out unpleasant feelings in communication with low-sociable people, can forgive, does not seek to re-educate those who do not like him(her), accepts habits and difficult character in other people.

This model of trust is characterized by empathy and a high degree of tolerance for almost all indicators. 
Consequently, a teacher who tends to trust in others, including students, even more than himself, is characterized by little self-trust, high levels of empathy and communicative tolerance.

In the total sample of teachers with such a model of communicative competence there proved to be the most $-20,5 \%$.

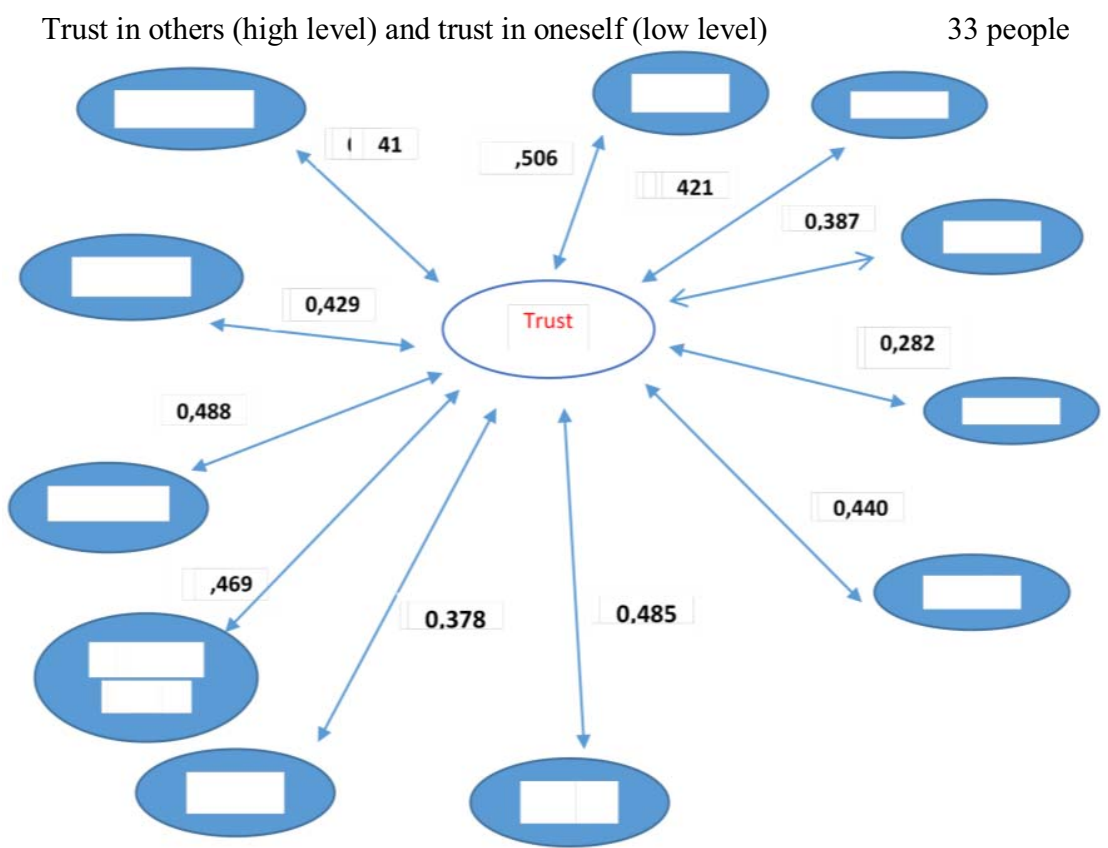

Scale 1. Rejection or misunderstanding of other person 's identity

Scale 2. Use of oneself as a benchmark in assessing behavior and way of thinking

Scale 3. Categorism or conservatism in assessing other people

Scale 4. Inability to hide or smooth unpleasant feelings when colliding with uncommunicative qualities of partners

Scale 5. Desire to change, re-educate partners

Scale 9. Inability to adapt to the character, habits and desires of others

Fig. 3. A model with high level of trust in others and low self-trust.

The picture changes somewhat when a teacher has a low level of trust in oneself and a high level of trust in others, that is, when a teacher tends to rely on others more than him(her)self in different situations. Here we have a large number of negative relationships, which include:

- adaptation

- avoidance

-rejection or misunderstanding of other person's identity

- use of oneself as a benchmark in assessing behaviour and way of thinking

- Categorism or conservatism in assessing other people

- Inability to hide or smooth unpleasant feelings when colliding with uncommunicative qualities of partners

- Desire to change, re-educate partners

- Desire to fit a partner to oneself, to make him(her) "convenient"

- Inability to adapt to the character, habits and desires of others.

Positive interrelations:

- cooperation 
- self-control

Positive relationships characterize him(her) as a person prone to cooperation at a high level of self-control. As seen from the qualities mentioned, a teacher with a low level of trust in oneself does not have relationship with empathy, but, alongside with this, does not accept adaptation and avoidance in a conflict situation, but prefers to strive for cooperation, and is prone to a high level of expression of communicative tolerance, which occurs in almost all parameters. It seems possible for us to suggest that a teacher with such a model of trust is trying to compensate low level of trust in oneself by seeking cooperation and empathy with other people.

In the total sample of teachers there proved to be $14 \%$ with such a model.

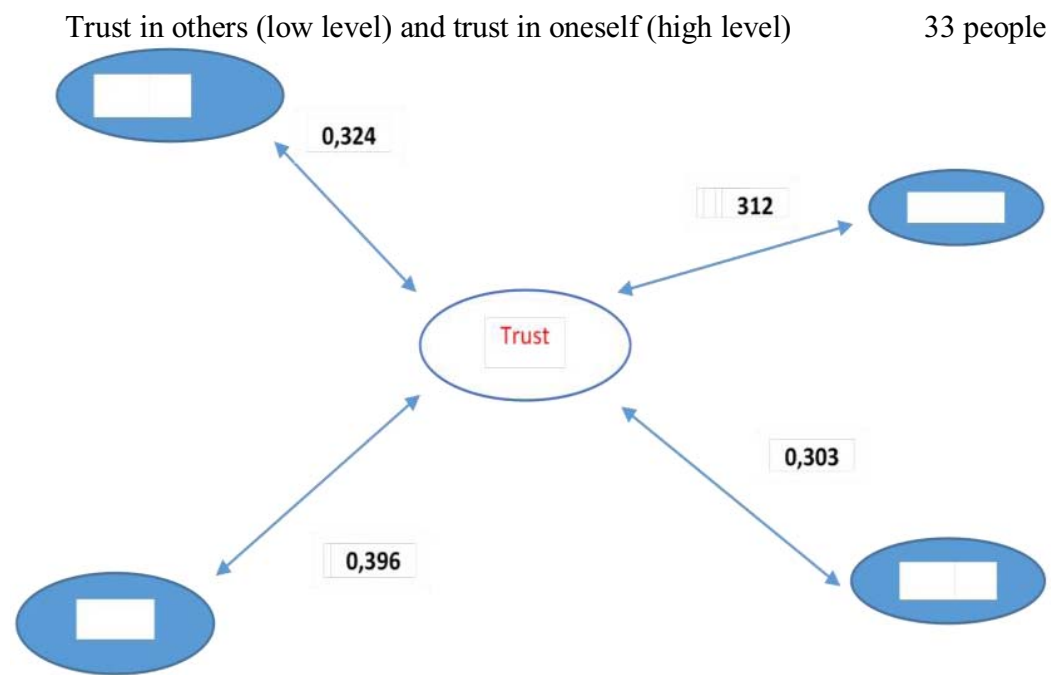

Scale 4. Inability to hide or smooth unpleasant feelings when colliding with uncommunicative qualities of partners

Scale 6. The desire to fit a partner, make him(her) "convenient"

Scale 7. Inability to forgive others ' mistakes, awkwardness, unintentional trouble caused to you.

Scale 9. Inability to adapt to the character, habits and desires of others

Fig. 4. A model with low level of trust in others and high self-trust.

As seen from the figure, this model contains a minimum number of relationships with other criteria of communicative competence. It is characterized by a high level of self-trust at the expense of that in others. Here we find the following relationships:

- Desire to fit a partner to oneself, to make him(her) "convenient";

- Inability to adapt to the character, habits and desires of others.

Negative interrelations:

- Inability to hide or smooth unpleasant feelings when colliding with uncommunitive qualities of partners

- Inability to forgive others ' mistakes, awkwardness, trouble caused to you inadvertently

This model is characterized by teacher 's desire not to adapt to a difficult interaction partner, but, on the contrary, to fit him(her), in other words, the desire to "break" a partner, to change him(her), he(she) does not consider necessary and does not seek to adapt to other people, to take into account their habits and desires, but at the same time, he(she) tries to forgive others their mistakes and miscalculations, and also seeks not to enter into conflict, in cases when he(she) has unpleasant feelings when communicating with others.

There proved to be quite a large number of teachers with such a model of trusting relations in the sample - $19 \%$. 


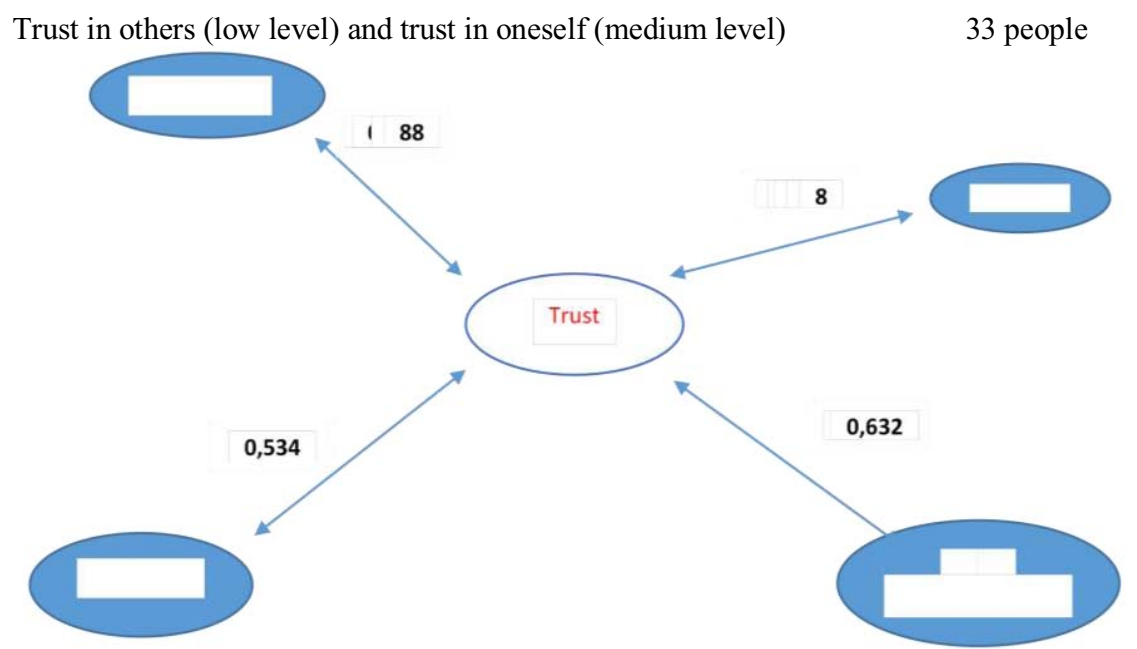

Scale 2. Use of oneself as a benchmark in assessing behavior and way of thinking

Fig. 5. A model with low trust in others and medium self-trust.

This model also has a small number of relationships with other parameters of communicative competence.

Positive interrelations:

- compromise

- Use itself as a benchmark in assessing behaviour and way of thinking.

Negative interrelations:

- adaptation

- self-control

This model is characterized by the fact that in interaction with other people a person quite often uses oneself as a standard, judges others by his(her) own standards, however, in a conflict situation seeks to find a compromise. Such a person is not inclined to adapt in a difficult conflict situation and does not bother him(her)self with high self-control, but prefers to behave spontaneously in any situation.

There proved to be $16 \%$ teachers with such a model of trust relations in the total sample. 


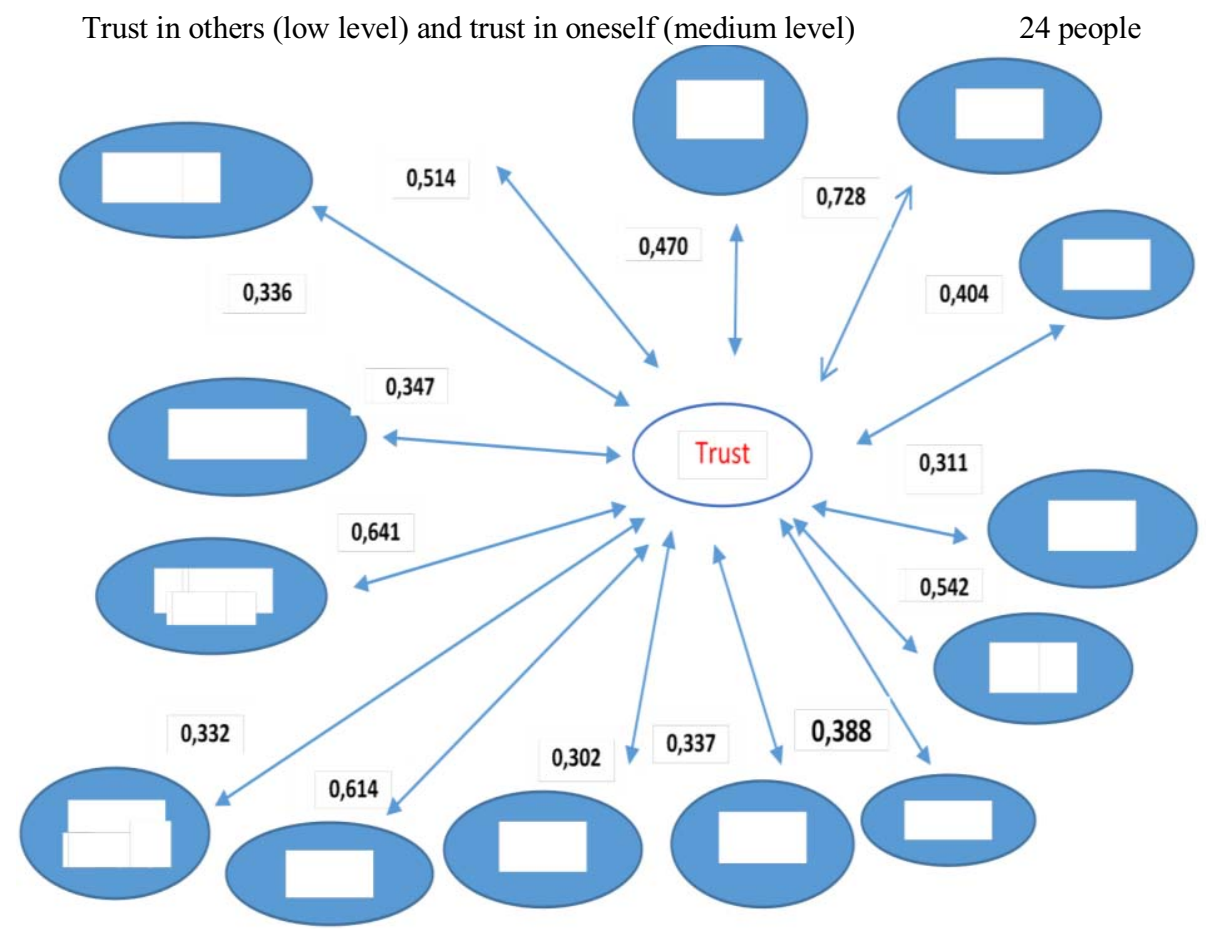

Scale 1. Rejection or misunderstanding of other person 's identity

Scale 2. Use of oneself as a benchmark in assessing behavior and way of thinking

Scale 3. Categorism or conservatism in assessing other people

Scale 4. Inability to hide or smooth unpleasant feelings when colliding with un-communicative qualities of partners

Scale 5. Desire to change, re-educate partners

Scale 6. The desire to fit a partner, make him(her) "convenient"

Scale 7. Inability to forgive others' mistakes, awkwardness, trouble caused to you inadvertently

Scale 8 . Intolerance of physical or mental discomfort created by others

Scale 9. Inability to adapt to the character, habits and desires of others

Fig. 6. A model with low self-trust and low level of trust in others.

A teacher with low-trust in oneself and others, has positive meaningful relationships with all scales of communicative tolerance, except scale 5, meaning the desire to change, reeducate partners, also positive relationships with virtually all other parameters of communicative competence.

Let us identify the positive and negative relationships that have emerged.

Positive ones:

rejection or misunderstanding of other person's identity

- use of oneself as a benchmark in assessing behaviour and way of thinking

- Categorism or conservatism in assessing other people

- Inability to hide or smooth unpleasant feelings when colliding with un-communicative qualities of partners

- Desire to fit the partner to oneself, to make him(her) "convenient"

- Inability to forgive others 'mistakes, awkwardness, trouble caused to you inadvertently

- Intolerance of physical or mental discomfort created by others

- Inability to adapt to the character, habits and desires of others

- empathy

- self-control 
- adaptation

Negative ones:

- Desire to change, re-educate partners

- compromise

- avoidance

At first glance, this model looks very contradictory. However, in our opinion, this contradiction can be interpreted as follows: a teacher with such a model of communicative competence has extreme intolerance towards people. He categorically does not trust them, however, he is able to hide his intolerance, since he has a tendency to adapt in difficult situations, and the ability to empathy can also be attributed here, apparently as one of the factors of the desire to adapt. It can be assumed that this type of teacher is prone to manipulation through the use of empathy and the desire for cooperation.

\section{Discussion}

So, a teacher with low-trust in oneself and others will show the following trends when interacting with others. First, such teachers do not bother themselves with such quality as communicative tolerance. They have positive relationships in almost all parameters, characterized as lack of communicative tolerance. Scale 5, meaning a desire to change and re-educate a partner, may mean that with such characteristics of trusting relationship teachers do not seek to somehow influence an interaction partner. Such teachers show extreme intolerance for what they do not like, it causes them irritation and intolerance. At the same time, they are able to mask his irritation with increased self-control in communication. They don't seek to join a conflict situation either, preferring to adapt to it. They will never compromise and even avoid in a conflict situation, they will prefer accommodation. Despite the lack of communicative tolerance in communication, at the same time they will never do what it takes, trying to change something in an interaction partner, which in general indicates their total indifference to others.

There proved to be the least - just over $10 \%$ of teachers with this model of communicative competence.

So, the analysis of the obtained models of communicative competence of modern teachers, based on the ratio of levels of trust in themselves and in others, showed that the most significant and evident parameter of communicative competence is communicative tolerance. The majority of modern teachers seek acceptance and understanding of students 'individuality, they, as a rule, do not seek to re-educate or change them considering individual personality qualities of students, they try not to use themselves as a benchmark in assessing students ' behaviour and way of thinking, part of them are not categorical in the assessment of students, they, as a rule, control themselves well when experiencing negative feelings and emotions in communicating with difficult students, know how to forgive mistakes and inadvertently caused troubles, tolerate discomfort, caused to them by other educational actors and also tend to adapt to the character and habits of students.

However, it should be noted that during the years of reform, empathy in interaction with students and also the desire for cooperation have lost their importance for most modern teachers. In complex, particularly tense situations, most teachers choose a strategy to adapt to difficult conditions.

It should also be noted that only $10 \%$ of modern teachers do not consider it necessary to show communicative tolerance in interaction with students. It seems that this part of teachers are very indifferent to their students, they do not care at all what others think about them, they are spontaneous, categorical, irritable and demanding of others, offensive and do not know how to forgive mistakes. 
However, at the same time, they are capable of empathy and self-control in interaction with students. But their main strategy of behavior is adaptation which, from our point of view, is related to indifference.

\section{Conclusion}

All in all, based on the compilation of the resulting models, a synthesis empirical model of a communicatively competent teacher can be constructed.

A communicatively competent teacher is a professional who is prone to tolerance in difficult situations, showing a high level of communicative tolerance, he is inclined to a strategy of compromise or adaptation in difficult situations, only a small number of teachers seek cooperation.

In our opinion, model No. 2, which is characterized by a high level, was the most communicatively competent model. The main characteristics of this model allow to build the following psychological portrait of such a teacher.

It is a teacher who is able to trust students at a high level, to show a high degree of empathy, which turns out to be a significant parameter for him in professional activity. This type of teacher is characterized by a high level of communication tolerance, which is expressed in the high degree of acceptance of students' individuality, lack of categorism in the evaluation of children, he(she) does not position him(her)self as a standard, can smooth out unpleasant feelings when communicating with low communicable and uncomfortable children, knows how to forgive, does not seek to re-educate those who do not like him, takes habits and difficult character in other people.

Thus, this model of trust is characterized by empathy and a high degree of tolerance for almost all indicators.

This model is based on a high level of trust in students, with a lower level of trust in their rightness and infallibility. However, as the analysis showed, there appeared slightly more than $20 \%$ of the total sample in our selection of teachers with such a set of qualities All other models obtained by us do not fully correspond to the ideas of communicative success of a teacher by different parameters.

\section{References}

1. A.I. Dontsov, E.B. Perelygina, O.Y. Zotova, S.V. Bridges, Social psychology and society 9(2), 21-34 (2018) doi: 10.17759/sps.2018090202

2. T.P. Skripkina, The european proceedings of social \& behavioural sciences epsbs, 651657 (2019) doi:10.15405 / epsbs.2019.07.86

3. S.N. Kostromina, S.D. Gurieva, M.M. Borisova, E.G. Khomyakova, Procedia - Social and Behavioral Sciences 23317, 35-41 (2016) doi: 10.1016/j.sbspro.2016.10.123

4. L. Teodora David, G. Lavinia Nită, Procedia - Social and Behavioral Sciences 12722, 139-143 (2014) doi: 10.1016/j.sbspro.2014.03.228

5. C.M. Corbin, P. Alamos, A.E. Lowenstein, JT. Downer, J.L. Brown, Journal of School Psychology 77, 1-12 (2019) DOI: 10.1016 / j. jsp.2019.10.001

6. A.L. Caruso, Mediterranean Journal of Clinical Psychology MJCP II(3) (2014) doi.org/10.6092/2282-1619/2014.2.1023

7. A.K. Mynbayeva, N. Anarbek, G.K. Akhmetova, Procedia - Social and Behavioral Sciences 6924, 1289-1294 (2012) doi: 10.1016/j.sbspro.2012.12.064 
8. K.T. Do, J.F. Guassi Moreira, E.H. Telzer, Developmental Cognitive Neuroscience 25, 260-271 (2017) https://doi.org/10.1016/j.dcn.2016.11.008

9. D. Türker, C. Altuntaş, Procedia - Social and Behavioral Sciences 150, 1177-1186 (2014) doi: 10.1016/j.sbspro.2014.09.133

10. E. Konya-Baumbach, M.C. Schuhmacher, S. Kuester, V. Kuharev, International Journal of Research in Marketing 36(3), 385-399 (2019) https://doi.org/10.1016/j.ijresmar.2019.01.008

11. A. Wullschleger, A. Garrote, S. Schnepel, L. Jaquiéry, E. Moser Opitz, Contemporary $\begin{array}{llll}\text { Educational Psychology } & \text { 60, } & 101841 & \text { (2020) }\end{array}$ https://doi.org/10.1016/j.cedpsych.2020.101841

12. T. Erkki Virtanen, G. S. Vaaland, S. Karin Ertesvåg, Teaching and Teacher Education 77, 240-252 (2019) https://doi.org/10.1016/j.tate.2018.10.013

13. D.M. Early, K.L. Maxwell, B.D. Ponder, Y. Pan, Early Childhood Research Quarterly 381, 57-70 (2017) http://dx.doi.org/10.1016/j.ecresq.2016.08.005

14. K. Aldrup, U. Klusmann, O. Lüdtke, R. Göllner, U. Trautwein, Learning and Instruction 58, 126-136 (2018) https://doi.org/10.1016/j.learninstruc.2018.05.006

15. Yaoxin Zhang, Wenxu Song, Zhenlin Tan, Huilin Zhu, Li Yi, Computers in Human Behavior 98, 140-149 (2019) https://doi.org/10.1016/j.chb.2019.04.008

16. L. Teodora David, G. Lavinia Nită, Procedia - Social and Behavioral Sciences 12722, 139-143 (2014) doi: 10.1016/j.sbspro.2014.03.228

17. B. Akçinar, Procedia - Social and Behavioral Sciences 9321, 1099-1104 (2013) doi: 10.1016/j.sbspro.2013.09.338

18. M. Keilow, C. Wu, C. Obel, SSM - Population Health 10, 100548 (2020) https://doi.org/10.1016/j.ssmph.2020.100548

19. I. Villanuevaa, M. Di Stefanob, L. Gellesa, P. Vicioso Osoriaa, S. Bensona, $\begin{array}{lllll}\text { Contemporary Educational } & \text { Psychology } & \text { 59, } & 101786 & \text { (2019) }\end{array}$ doi.org/10.1016/j.cedpsych.2019.101786

20. R.E. Lacey, S.M. Pinto Pereira, L. Li, A. Danese, Brain, Behavior, and Immunityn (2020) https://doi.org/10.1016/j.bbi.2020.03.017

21. T.P. Skripkina, The european proceedings of social \& behavioural sciences epsbs, 651657 (2019) doi:10.15405 / epsbs.2019.07.86

22. S.A.C. Pangalila, Y. Budiarto, Humanitas 14(2), $150 \quad-163$ (2017) https://www.researchgate.net/publication/330108542_Factor_analysis_of_Rotter's_Inte rpersonal_trust_scale

23. Psychometric method "self-Confidence" by Mark Snyder, https://www.psychometrica.ru/index.php/metodiks/lerningbook-methods-1

24. V.V. Boyko, Psychoenergetics (Peter, SPb, 2008) ISBN 978-5-91180-760-3 https://bookap.info/okolopsy/boyko_psihoenergetika/

25. K.J. Michalska, K.D. Kinzler, Jean Decety, Developmental Cognitive Neuroscience 3, 22-32 (2013) http://dx.doi.org/10.1016/j.den.2012.08.001

26. I. Petersen, I. Douglas, H. Whitaker, BMJ 354 (2016) doi: https://doi.org/10.1136/bmj.i4515

27. R.H. Kilmann, K.W. Thomas, Educational and Psychological Measurement 37(2), 309325

https://www.researchgate.net/publication/247726333_Developing_a_ForcedChoice_Measure_of_Conflict-Handling_Behavior_The_Mode_Instrument 